\title{
The Nonexistence of Global Solutions for a Time Fractional Schrödinger Equation with Nonlinear Memory
}

\author{
Yaning Li ${ }^{*}$, Quanguo Zhang² \\ ${ }^{1}$ College of Mathematics \& Statistics, Nanjing University of Information Science \& Technology, Nanjing, China \\ ${ }^{2}$ Department of Mathematics, Luoyang Normal University, Luoyang, Henan, China \\ Email: *liyn11@126.com, zhangqg07@163.com
}

How to cite this paper: Li, Y.N. and Zhang, Q.G. (2018) The Nonexistence of Global Solutions for a Time Fractional Schrödinger Equation with Nonlinear Memory. Journal of Applied Mathematics and Physics, 6, 1418-1424. https://doi.org/10.4236/jamp.2018.67118

Received: June 7, 2018

Accepted: July 7, 2018

Published: July 10, 2018

Copyright ( 92018 by authors and Scientific Research Publishing Inc. This work is licensed under the Creative Commons Attribution International License (CC BY 4.0).

http://creativecommons.org/licenses/by/4.0/

\section{cc) (i) Open Access}

\begin{abstract}
In this paper, we study the nonexistence of solutions of the following time fractional nonlinear Schrödinger equations with nonlinear memory

$$
\left\{\begin{array}{l}
i^{\alpha}{ }_{0}^{C} D_{t}^{\alpha} u+\Delta u=\lambda_{0} I_{t}^{1-\gamma}\left(|u|^{p}\right), x \in \mathbb{R}^{N}, t>0, \\
u(0, x)=g(x), \quad x \in \mathbb{R}^{N},
\end{array}\right.
$$

where $0<\alpha<\gamma<1, i^{\alpha}$ denotes the principal value of $i^{\alpha}, p>1, T>0$, $\lambda \in \mathbb{C} \backslash\{0\}, u(t, x)$ is a complex-value function, ${ }_{0} I_{t}^{1-\gamma}$ denotes left Riemann-Liouville fractional integrals of order $1-\gamma$ and ${ }_{0}^{C} D_{t}^{\alpha} u$ is the Caputo fractional derivative of order $\alpha$. We obtain that the problem admits no global weak solution when $1<p<1+\frac{2(\alpha+1-\gamma)}{\alpha N}$ and $1<p<1+\frac{1-\gamma}{\alpha}$ under different conditions for initial data.

\section{Keywords}

Fractional Schrödinger Equation, Nonexistence, Cauchy Problems, Nonlinear Memory
\end{abstract}

\section{Introduction}

This paper is concerned with the nonexistence of solutions to the Cauchy problem for the time fractional nonlinear Schrödinger equations with nonlinear memory

$$
\left\{\begin{array}{l}
i_{0}^{\alpha}{ }_{0}^{C} D_{t}^{\alpha} u+\Delta u=\lambda_{0} I_{t}^{1-\gamma}\left(|u|^{p}\right), x \in \mathbb{R}^{N}, t>0, \\
u(0, x)=g(x), \quad x \in \mathbb{R}^{N},
\end{array}\right.
$$


where $0<\alpha<\gamma<1, i^{\alpha}$ denotes principal value of $i^{\alpha}, \quad p>1, T>0$, $\lambda=\lambda_{1}+\lambda_{2} i \in \mathbb{C} \backslash\{0\}, \lambda_{1}, \lambda_{2} \in \mathbb{R}, u=u(t, x)$ is a complex-valued function, $g(x)=g_{1}(x)+g_{2}(x) i, \quad g_{1}(x)$ and $g_{2}(x)$ are real-valued functions. ${ }_{0} I_{t}^{1-\gamma}$ denotes left Riemann-Liouville fractional integrals of order $1-\gamma$ and

$$
{ }_{0}^{C} D_{t}^{\alpha} u=\frac{\partial}{\partial t}{ }_{0} I_{t}^{1-\alpha}(u(t, x)-u(0, x)) \text {. }
$$

For the nonlinear Schrödinger equations without gauge invariance (i.e. $\alpha=\gamma=1$ ),

$$
\left\{\begin{array}{l}
i u_{t}+\Delta u=\lambda|u|^{p}, x \in \mathbb{R}^{N}, t>0 \\
u(0, x)=g(x), x \in \mathbb{R}^{N}
\end{array}\right.
$$

Ikeda and Wakasugi [1] and Ikeda and Inui [2] [3] proved blow-up results of solutions for (2) under different conditions for

$$
1<p<1+\frac{2}{N} \text { and } 1<p<1+\frac{4}{N} \text {. }
$$

The main tool they used is test function method. This method is based on rescalings of a compactly support test function to prove blow-up results which is first used by Mitidieri and Pohozaev [4] to show the blow-up results.

Recently, it has been seen that fractional differential equations have better effects in many realistic applications than the classical ones. So, considerable attention has been attracted to time fractional diffusion equation which arises in electromagnetic, acoustic and mechanical phenomena etc. [5], and is derived from classical diffusion equation by replacing the first-order time derivative by a fractional derivative of order $\alpha$ with $\alpha \in(0,1]$. Fractional diffusion equation was explicitly applied to physics by Nigmatullin [6] to describe diffusion in media with fractal geometry (special types of porous media). There are many papers about the existence and properties of solutions for fractional differential equation, see for example [7] [8] [9] [10] [11] and the references therein.

For nonlinear time fractional Schrödinger equations (i.e., (1) with $\gamma=1$ ), Zhang, Sun and Li [12] studied the nonexistence of this problem in $C_{0}\left(R^{N}\right)$ and proved that the problem admits no global weak solution with suitable initial data when $1<p<1+\frac{2}{N}$ by using test function method, and also give some conditions which imply the problem has no global weak solution for every $p>1$.

In [13], Cazenave, Dickstein and Weissler considered a class of heat equation with nonlinear memory. They obtained that the solution blows up in finite time and under suitable conditions the solution exists globally. In [14], using test function method, the authors considered a heat equation with nonlinear memory, they determined Fujita critical exponent of the problem.

Motivated by above results, in present paper, our purpose is to study the nonexistence of global weak solutions of (1) with a condition related to the sign of initial data when 


$$
1<p<1+\frac{2(\alpha+1-\gamma)}{\alpha N} \text { and } 1<p<1+\frac{1-\gamma}{\alpha} .
$$

This paper is organized as follows. In Section 2, some preliminaries and the main results are presented. In Section 3, we give proof of the main results.

\section{Preliminaries and the Main Results}

For convenience of statement, let us present some preliminaries that will be used in next sections.

If ${ }_{0}^{C} D_{t}^{\alpha} f \in L^{1}(0, T), g \in C^{1}([0, T])$ and $g(T)=0$, then we have the following formula of integration by parts

$$
\int_{0}^{T} g_{0}^{C} D_{t}^{\alpha} f \mathrm{~d} t=\int_{0}^{T}(f(t)-f(0)){ }_{t}^{C} D_{T}^{\alpha} g \mathrm{~d} t .
$$

We need calculate Caputo fractional derivative of the following function, which will be used in next sections. For given $T>0$ and $n>0$, if we let

$$
\varphi(t)= \begin{cases}\left(1-\frac{t}{T}\right)^{n}, & t \leq T, \\ 0, & t>T,\end{cases}
$$

then

$$
{ }_{t}^{C} D_{T}^{\alpha} \varphi(t)=\frac{\Gamma(n+1)}{\Gamma(n+1-\alpha)} T^{-\alpha}\left(1-\frac{t}{T}\right)^{n-\alpha}, t \leq T,
$$

(see for example [15]).

Now, we present the definition of weak solution of (1).

Definition 2.1. Let $g \in L_{\text {loc }}^{1}\left(R^{N}\right), \quad 0<\alpha<\gamma<1$ and $T>0$, we call $u \in L^{p}\left((0, T), L_{l o c}^{\infty}\left(R^{N}\right)\right)$ is a weak solution of (1) if

$$
\int_{R^{N}} \int_{0}^{T} \lambda_{0} I_{t}^{1-\gamma}\left(|u|^{p}\right) \varphi+i^{\alpha} g(x){ }_{t}^{C} D_{T}^{\alpha} \varphi \mathrm{d} t \mathrm{~d} x=\int_{R^{N}} \int_{0}^{T} u\left(\Delta \varphi+i^{\alpha}{ }_{t}^{C} D_{T}^{\alpha} \varphi\right) \mathrm{d} t \mathrm{~d} x
$$

for every $\varphi \in C_{x, t}^{2,1}\left(R^{N} \times[0, T]\right) \quad$ with $\quad \operatorname{supp}_{x} \varphi \subset \subset R^{N} \quad$ and $\quad \varphi(x, T)=0$. Moreover, if $T>0$ can be arbitrarily chosen, then we call $u$ is a global weak solution for of (1).

Denote

$$
G_{1}(x)=\cos \frac{\pi \alpha}{2} g_{1}(x)-\sin \frac{\pi \alpha}{2} g_{2}(x), G_{2}(x)=\cos \frac{\pi \alpha}{2} g_{2}(x)+\sin \frac{\pi \alpha}{2} g_{1}(x)
$$

and $\beta=1-\gamma$.

The following theorems show main result of this paper.

Theorem 2.2. Let $1<p<1+\frac{2(\alpha+\beta)}{\alpha N}$. If $g \in L^{1}\left(\mathbb{R}^{N}\right)$ and satisfies

$$
\lambda_{1} \int_{\mathbb{R}^{N}} G_{1}(x) \mathrm{d} x>0 \text {, or } \lambda_{2} \int_{\mathbb{R}^{N}} G_{2}(x) \mathrm{d} x>0,
$$

then problem (1) admits no global weak solution.

Theorem 2.3. If $1<p<1+\frac{\beta}{\alpha}$, let $\chi(x)=\left(\int_{\mathbb{R}^{N}} \mathrm{e}^{-\sqrt{N^{2}+|x|^{2}}} \mathrm{~d} x\right)^{-1} \mathrm{e}^{-\sqrt{N^{2}+|x|^{2}}}$. If 
$g \in L_{\left(\mathbb{R}^{N}\right)}^{1}$ and satisfies

$$
\lambda_{1} \int_{\mathbb{R}^{N}} G_{1}(x) \chi(x) \mathrm{d} x>0 \text {, or } \lambda_{2} \int_{\mathbb{R}^{N}} G_{2}(x) \chi(x) \mathrm{d} x>0,
$$

then problem (1) admits no global weak solution.

\section{Proofs of Main Result}

In this section, we prove blow-up results and global existence of mild solutions of (1).

Proof of Theorem 2.2. If

$$
1<p<1+\frac{2(\alpha+\beta)}{\alpha N},
$$

for the case $\lambda_{1} \int_{\mathbb{R}^{N}} G_{1}(x) \mathrm{d} x>0$, we may as well suppose that $\lambda_{1}>0$ and $\int_{\mathbb{R}^{N}} G_{1}(x) \mathrm{d} x>0$. Let $\Phi \in C_{0}^{\infty}\left(\mathbb{R}^{N}\right)$ such that $\Phi(s)=1$ for $|s| \leq 1, \Phi(s)=0$ for $|s|>2$ and $0 \leq \Phi(s) \leq 1$. For $T>0$, we define

$$
\varphi_{1}(x)=\left(\Phi\left(T^{-\frac{\alpha}{2}}|x|\right)\right)^{\frac{2 p}{p-1}}, \varphi_{2}(t)=\left(1-\frac{t}{T}\right)^{m}, m \geq \max \left\{1, \frac{p(\alpha+\beta)}{p-1}\right\}, t \in[0, T] .
$$

Let $\varphi(x, t)={ }_{t}^{C} D_{T}^{\beta} \varphi_{1}(x) \varphi_{2}(t)$. Assuming that $u$ is a weak solution of (1), and since $\alpha+\beta<1$, we have

$$
\begin{aligned}
& R e \int_{\mathbb{R}^{N}} \int_{0}^{T} \lambda|u|^{p} \varphi_{1} \varphi_{2}+i^{\alpha} g(x) \varphi_{1}{ }_{t}^{C} D_{T}^{\alpha+\beta} \varphi_{2} \mathrm{~d} t \mathrm{~d} x \\
& =\operatorname{Re} \int_{\mathbb{R}^{N}} \int_{0}^{T} u\left(\Delta \varphi_{1}\right){ }_{t}^{C} D_{T}^{\beta} \varphi_{2}+i^{\alpha} u \varphi_{1}{ }_{t}^{C} D_{T}^{\alpha+\beta} \varphi_{2} \mathrm{~d} t \mathrm{~d} x .
\end{aligned}
$$

that is

$$
\begin{aligned}
& \int_{\mathbb{R}^{N}} \int_{0}^{T} \lambda_{1}|u|^{p} \varphi_{1} \varphi_{2}+\left(\cos \frac{\pi \alpha}{2} g_{1}-\sin \frac{\pi \alpha}{2} g_{2}\right) \varphi_{1}{ }_{t}^{C} D_{T}^{\alpha+\beta} \varphi_{2} \mathrm{~d} t \mathrm{~d} x \\
& =\int_{\mathbb{R}^{N}} \int_{0}^{T}(R e u)\left(\Delta \varphi_{1}\right){ }_{t}^{C} D_{T}^{\beta} \varphi_{2}+\left(\cos \frac{\pi \alpha}{2} R e u-\sin \frac{\pi \alpha}{2} \operatorname{Imu}\right) \varphi_{1}{ }_{t}^{C} D_{T}^{\alpha+\beta} \varphi_{2} \mathrm{~d} t \mathrm{~d} x \\
& \leq \int_{\mathbb{R}^{N}} \int_{0}^{T}|u|\left(\left(\Delta \varphi_{1}\right){ }_{t}^{C} D_{T}^{\beta} \varphi_{2}+\varphi_{1}{ }_{t}^{C} D_{T}^{\alpha+\beta} \varphi_{2}\right) \mathrm{d} t \mathrm{~d} x .
\end{aligned}
$$

Note that

$$
\left|\left(\Delta \varphi_{1}\right){ }_{t}^{C} D_{T}^{\beta} \varphi_{2}+\varphi_{1}^{C}{ }_{t}^{C} D_{T}^{\alpha+\beta} \varphi_{2}\right| \leq C T^{-(\alpha+\beta)} \varphi_{1}^{\frac{1}{p}} \varphi_{2}^{\frac{1}{p}}
$$

for some positive constant $C$ independent of $T$. Then, by (4), (5) and Hölder inequality, we have

$$
\begin{aligned}
& \lambda_{1} \int_{\mathbb{R}^{N}} \int_{0}^{T}|u|^{p} \varphi_{1} \varphi_{2} \mathrm{~d} t \mathrm{~d} x+\int_{R^{N}} \int_{0}^{T}\left(\cos \frac{\pi \alpha}{2} g_{1}-\sin \frac{\pi \alpha}{2} g_{2}\right) \varphi_{1}{ }_{t}^{C} D_{T}^{\alpha+\beta} \varphi_{2} \mathrm{~d} t \mathrm{~d} x \\
& \leq C T^{-(\alpha+\beta)} \int_{\mathbb{R}^{N}} \int_{0}^{T}|u| \varphi_{1}^{\frac{1}{p}} \varphi_{2}^{\frac{1}{p}} \mathrm{~d} x \mathrm{~d} t \\
& \leq C T^{-(\alpha+\beta)+\left(1+\frac{\alpha N}{2}\right) \frac{p-1}{p}}\left(\int_{\mathbb{R}^{N}} \int_{0}^{T}|u|^{p} \varphi_{1} \varphi_{2} \mathrm{~d} x \mathrm{~d} t\right)^{\frac{1}{p}} \\
& \leq C T^{1+\frac{\alpha N}{2}-\frac{p(\alpha+\beta)}{p-1}}+\frac{\lambda_{1}}{2} \int_{\mathbb{R}^{N}} \int_{0}^{T}|u|^{p} \varphi_{1} \varphi_{2} \mathrm{~d} t \mathrm{~d} x .
\end{aligned}
$$


Hence

$$
T^{1-\alpha-\beta} \int_{\mathbb{R}^{N}} G_{1}(x) \varphi_{1} \mathrm{~d} x \leq C T^{1+\frac{\alpha N}{2}-\frac{p(\alpha+\beta)}{p-1}} .
$$

Since $p<1+\frac{2(\alpha+\beta)}{\alpha N}$, we have $\alpha+\beta+\frac{\alpha N}{2}-\frac{p(\alpha+\beta)}{p-1}<0$. Therefore, if the solution of (1) exists globally, then taking $T \rightarrow \infty$, we obtain

$$
\int_{\mathbb{R}^{N}} G_{1}(x) \mathrm{d} x \leq 0,
$$

which contradicts with the assumption.

For case $\lambda_{2} \int_{\mathbb{R}^{N}} G_{2}(x) \mathrm{d} x>0$, we have

$$
\begin{aligned}
& \operatorname{Im} \int_{\mathbb{R}^{N}} \int_{0}^{T} \lambda|u|^{p} \varphi_{1} \varphi_{2}+i^{\alpha} g(x) \varphi_{1}{ }_{t}^{C} D_{T}^{\alpha+\beta} \varphi_{2} \mathrm{~d} x \mathrm{~d} t \\
& =\operatorname{Im} \int_{\mathbb{R}^{N}} \int_{0}^{T} u\left(\Delta \varphi_{1}\right){ }_{t}^{C} D_{T}^{\beta} \phi_{2}+i^{\alpha} u \varphi_{1}{ }_{t}^{C} D_{T}^{\alpha+\beta} \varphi_{2} \mathrm{~d} x \mathrm{~d} t .
\end{aligned}
$$

Then by a similar proof as above, we can also obtain a contradiction.

Proof of Theorem 2.3. We only consider the case $\lambda_{1}>0$ and $\int_{\mathbb{R}^{N}} G_{1}(x) \chi(x) \mathrm{d} x>0$, since other cases can be proved by a similar method. Take $\psi \in C_{0}^{\infty}\left(\mathbb{R}^{N}\right)$ such that

$$
\psi(x)= \begin{cases}1, & |x| \leq 1 \\ 0, & |x| \geq 2\end{cases}
$$

and $0 \leq \psi(x) \leq 1, \quad x \in \mathbb{R}^{N}$. Let $\psi_{n}(x)=\psi\left(\frac{x}{n}\right), n=1,2, \cdots$. Suppose that $u$ is a bounded weak solution of (1), taking

$$
\varphi_{1}(x)=\chi(x) \psi_{n}(x), \varphi_{2}(t)=\left(1-\frac{t}{T}\right)^{m}, m>\max \left\{2, \frac{p(\alpha+\beta)}{p-1}\right\},
$$

and define $\varphi(x, t)={ }_{t}^{C} D_{T}^{\beta} \varphi_{1}(x) \varphi_{2}(t)$, then using the definition of weak solution of (1) and since $\alpha+\beta<1$, we derive that

$$
\begin{aligned}
& \operatorname{Re} \int_{\mathbb{R}^{N}} \int_{0}^{T} \lambda|u|^{p} \varphi_{1} \varphi_{2}+i^{\alpha} g(x) \varphi_{1}{ }_{t}^{C} D_{T}^{\alpha+\beta} \varphi_{2} \mathrm{~d} x \mathrm{~d} t \\
& =\operatorname{Re} \int_{\mathbb{R}^{N}} \int_{0}^{T} u\left(\Delta \varphi_{1}\right){ }_{t}^{C} D_{T}^{\beta} \varphi_{2}+i^{\alpha} u \varphi_{1}{ }_{t}^{C} D_{T}^{\alpha+\beta} \varphi_{2} \mathrm{~d} x \mathrm{~d} t .
\end{aligned}
$$

Since

$$
\Delta \varphi_{1}=\Delta\left(\chi \psi_{n}\right)=(\Delta \chi) \psi_{n}+2 \nabla \chi \cdot \nabla \psi_{n}+\left(\Delta \psi_{n}\right) \chi
$$

and

$$
|\Delta \chi|=\left(-\frac{N}{\sqrt{N^{2}+|x|^{2}}}+\frac{x^{2}}{N^{2}+|x|^{2}}+\frac{|x|^{2}}{\left(N^{2}+|x|^{2}\right)^{\frac{3}{2}}}\right) \leq 3 \chi,
$$

by (6) and dominated convergence theorem, let $n \rightarrow \infty$, we have

$$
\begin{aligned}
& \int_{\mathbb{R}^{N}} \int_{0}^{T} \lambda_{1}|u|^{p} \chi \varphi_{2}+\chi\left(\cos \frac{\pi \alpha}{2} g_{1}(x)-\sin \frac{\pi \alpha}{2} g_{2}(x)\right){ }_{t}^{C} D_{T}^{\alpha+\beta} \varphi_{2} \mathrm{~d} x \mathrm{~d} t \\
& \leq \int_{\mathbb{R}^{N}} \int_{0}^{T} 3|u| \chi_{t}^{C} D_{T}^{\beta} \varphi_{2}+\chi\left(\cos \frac{\pi \alpha}{2} R e u-\sin \frac{\pi \alpha}{2} \operatorname{Imu}\right){ }_{t}^{C} D_{T}^{\alpha+\beta} \varphi_{2} \mathrm{~d} x \mathrm{~d} t .
\end{aligned}
$$


Hence, by Jensen's inequality and (7), we have

$$
\begin{aligned}
& \lambda_{1} \int_{0}^{T}\left(\int_{\mathbb{R}^{N}}|u| \chi\right)^{p} \varphi_{2} \mathrm{~d} t d x+\int_{\mathbb{R}^{N}} \int_{0}^{T} \chi\left(\cos \frac{\pi \alpha}{2} g_{1}(x)-\sin \frac{\pi \alpha}{2} g_{2}(x)\right){ }_{t}^{C} D_{T}^{\alpha+\beta} \varphi_{2} \mathrm{~d} t \mathrm{~d} x \\
& \leq \int_{\mathbb{R}^{N}} \int_{0}^{T} 3|u| \chi{ }_{t}^{C} D_{T}^{\beta} \varphi_{2}+\chi|u|{ }_{t}^{C} D_{T}^{\alpha+\beta} \varphi_{2} \mathrm{~d} x \mathrm{~d} t .
\end{aligned}
$$

Denoting $f(t)=\int_{\mathbb{R}^{N}}|u| \chi \mathrm{d} x$, and $A=\int_{\mathbb{R}^{N}} \chi\left(\cos \frac{\pi \alpha}{2} g_{1}(x)-\sin \frac{\pi \alpha}{2} g_{2}(x)\right) \mathrm{d} x$, then we have

$$
\begin{aligned}
& \lambda_{1} \int_{0}^{T} f^{p} \varphi_{2} \mathrm{~d} t+A \int_{0}^{T^{C}} D_{T}^{\alpha+\beta} \varphi_{2} \mathrm{~d} t \\
& \leq 3 \int_{0}^{T} f^{C} D_{T}^{\beta} \varphi_{2} \mathrm{~d} t+\int_{0}^{T} f^{C} D_{T}^{\alpha+\beta} \varphi_{2} \mathrm{~d} t \\
& =3 \int_{0}^{T} f \varphi_{2}^{\frac{1}{p}} \varphi_{2}^{-\frac{1}{p}}{ }_{t}^{C} D_{T}^{\beta} \varphi_{2} \mathrm{~d} t+\int_{0}^{T} f \varphi_{2}^{\frac{1}{p}} \varphi_{2}^{-\frac{1}{p}}{ }_{t}^{C} D_{T}^{\alpha+\beta} \varphi_{2} \mathrm{~d} t \\
& \leq \frac{\lambda_{1}}{2} \int_{0}^{T} f^{p} \varphi_{2} \mathrm{~d} t+C \int_{0}^{T} \varphi_{2}^{-\frac{1}{p-1}}\left({ }_{t}^{C} D_{T}^{\beta} \varphi_{2}\right)^{\frac{p}{p-1}} \mathrm{~d} t+C \int_{0}^{T} \varphi_{2}^{-\frac{1}{p-1}}\left({ }_{t}^{C} D_{T}^{\alpha+\beta} \varphi_{2}\right)^{\frac{p}{p-1}} \mathrm{~d} t .
\end{aligned}
$$

Thus,

$$
\frac{\lambda_{1}}{2} \int_{0}^{T} f^{p} \varphi_{2} \mathrm{~d} t+C A T^{1-\alpha-\beta} \leq C\left(T^{1-\frac{p \beta}{p-1}}+T^{1-\frac{p(\alpha+\beta)}{p-1}}\right)
$$

So,

$$
A \leq C\left(T^{\alpha+\beta-\frac{p \beta}{p-1}}+T^{\frac{-(\alpha+\beta)}{p-1}}\right)
$$

since $p<1+\frac{\beta}{\alpha}$, we get $A \leq 0$ by taking $T \rightarrow \infty$, which contradicts with the assumption. Therefore, if $u \in C\left([0, T) \times \mathbb{R}^{N} ; R\right)$ is a solution of (1), then $T<+\infty$.

\section{Supported}

Supported by NSF of China $(11626132,11601216)$.

\section{References}

[1] Ikeda, M. and Wakasugi, Y. (2013) Small Data Blow-Up of $L^{2}$-Solution for the Nonlinear Schrödinger Equation without Gauge Invariance. Differential Integral Equations, 26, 1275-1285.

[2] Ikeda, M. and Inui, T. (2015) Small Data Blow-Up of $L^{2}$ or $H^{1}$-Solution for the Semilinear Schrodinger Equation without Gauge Invariance. Journal of Evolution Equations, 15, 1-11. https://doi.org/10.1007/s00028-015-0273-7

[3] Ikeda, M. and Inui, T. (2015) Some Non-Existence Results for the Semilinear Schrödinger Equation without Gauge Invariance. Journal of Mathematical Analysis and Applications, 425, 758-773. https://doi.org/10.1016/j.jmaa.2015.01.003

[4] Mitidieri, E. and Pohozaev, S.I. (2001) A Priori Estimates and Blow-Up of Solutions to Nonlinear Partial Differential Equations and Inequalities. Proceedings of the Steklov Institute of Mathematics, 234, 1-383. 
[5] Mainardi, F. (1994) On the Initial Value Problem for the Fractional Diffusion-Wave Equation. In Rionero, S. and Ruggeri, T., Eds., Waves and Stability in Continuous Media, World Scientific, Singapore, 246-251.

[6] Nigmatullin, R.R. (1986) The Realization of the Generalized Transfer Equation in a Medium with Fractal Geometry. Physica Status Solidi, 133, 425-430.

https://doi.org/10.1002/pssb.2221330150

[7] Andrade, B. and Viana, A. (2017) On a Fractional Reaction-Diffusion Equation. Zeitschrift für angewandte Mathematik und Physik, 68, 59. https://doi.org/10.1007/s00033-017-0801-0

[8] Li, Y.N. (2015) Regularity of Mild Solutions for Fractional Abstract Cauchy Problem with Order $\alpha \in(1,2)$. Zeitschrift für angewandte Mathematik und Physik, 66, 3283-3298. https://doi.org/10.1007/s00033-015-0577-Z

[9] Li, Y.N., Sun, H.R. and Feng, Z.S. (2016) Fractional Abstract Cauchy Problem with Order $\alpha \in(1,2)$. Dynamics of PDE, 13, 155-177.

[10] Vergara, V. and Zacher, R. (2017) Stability, Instability, and Blowup for Time Fractional and Other Nonlocal in Time Semilinear Subdiffusion Equations. Journal of Evolution Equations, 17, 599-626. https://doi.org/10.1007/s00028-016-0370-2

[11] Zhang, Q.G. and Sun, H.R. (2015) The Blow-Up and Global Existence of Solutions of Cauchy Problems for a Time Fractional Diffusion Equation. Topological Methods in Nonlinear Analysis, 46, 69-92. https://doi.org/10.12775/TMNA.2015.038

[12] Zhang, Q.G., Sun, H.R. and Li, Y.N. (2017) The Nonexistence of Global Solutions for a Time Fractional Nonlinear Schrödinger Equation without Gauge Invariance. Applied Mathematics Letters, No. 64, 119-124. https://doi.org/10.1016/j.aml.2016.08.017

[13] Cazenave, T., Dickstein, F. and Weissler, F.B. (2008) An Equation Whose Fujita Critical Exponent Is Not Given by Scaling. Nonlinear Analysis, 68, 862-874. https://doi.org/10.1016/j.na.2006.11.042

[14] Fino, A.Z. and Kirane, M. (2012) Qualitative Properties of Solutions to a Time-Space Fractional Evolution Equation. Quarterly of Applied Mathematics, 70, 133-157. https://doi.org/10.1090/S0033-569X-2011-01246-9

[15] Kilbas, A.A., Srivastava, H.M. and Trujillo, J.J. (2006) Theory and Applications of Fractional Differential Equations, Vol 204. Elsevier Science B.V., Amsterdam. https://doi.org/10.1016/S0304-0208(06)80001-0 